\title{
Daily life activity in patients with left ventricular assist devices
}

\author{
M. Granegger ${ }^{1,2}$, T. Schlöglhofer ${ }^{1,2,3}$, H. Ober ${ }^{1}$, D. Zimpfer ${ }^{3}$, H. Schima ${ }^{1,2,3}$, and F. \\ Moscato $^{1,2}$ \\ ${ }^{1}$ Center for Medical Physics and Biomedical Engineering, Medical University of Vienna, Vienna, \\ Austria \\ ${ }^{2}$ Ludwig-Boltzmann-Cluster for Cardiovascular Research, Vienna, Austria \\ ${ }^{3}$ Department of Cardiac Surgery, Medical University of Vienna, Vienna, Austria
}

\section{Abstract}

Exercise capacity is usually evaluated by peak oxygen consumption (peak- $\left.\mathrm{VO}_{2}\right)$. However, assessment of peak- $\mathrm{VO}_{2}$ in patients with a left ventricular assist device (LVAD) might not be the best method to provide insight into their daily life activity. Aim of this study was to assess postoperative activity of LVAD patients using actigraphy and to compare these patients to a healthy and a heart transplanted (HTx) population.

Activity was continuously monitored using wrist-accelerometers in LVAD patients after implantation, during 4 weeks of rehabilitation following hospital discharge, and at two follow-up assessments. Peak- $\mathrm{VO}_{2}$ values measured during rehabilitation were correlated with activity. Additionally, actigraphy data from LVAD recipients were compared with data measured in healthy and HTx subjects.

After hospital discharge a significant increase in physical activity of LVAD recipients was observed ( $55 \pm 28$ vs. $102 \pm 23$ Activity Scores, $\mathrm{n}=11, \mathrm{p}=0.002$ ). During rehabilitation, as well as at the follow-ups ( $140 \pm 43$ and $253 \pm 33$ days post implantation) no significant increase in activity was observed. Peak- $\mathrm{VO}_{2}$ values correlated to daily activity both in LVAD and HTx patients $(\mathrm{r}>0.5)$. Average daily activity was significantly lower in LVAD and HTx patients than in the healthy population ( $130 \pm 30$ and $148 \pm 60$ vs. $245 \pm 63$ Activity Score; $n=18$ in each group, $p<0.001$ ).

Activity in LVAD recipients increased substantially after hospital discharge with no further significant improvement observed during a period of 8.5 months. Similarly to the peak- $\mathrm{VO}_{2}$, also the daily activity of LVAD recipients was $53 \%$ compared to healthy subjects. These results highlight the need for an optimized physical therapy in this patient cohort.

\section{Introduction}

Recently, continuous flow left ventricular assist devices (LVADs) became the therapy of choice for patients with terminal cardiac insufficiency. Due to the success of these systems, the area of application changed: in the past they were used as bridge to transplant; now they

Discplosures

Conflict of interest: None. 
are also used for life-time as destination therapy (1). Therefore, the preservation of the residual native cardiac function as well as the potential cardiac and overall physical recovery come into focus (2). To improve the treatment of patients with an LVAD implanted, assessment of the overall physical condition of the patients might be of major importance as it is the case in heart transplanted (HTx) patients. In this latter patient population the positive impact obtained by appropriate training and rehabilitation is known (3), with an increased peak oxygen consumption (peak- $\mathrm{VO}_{2}$ ) being identified as a prognostic factor for survival (3). In case of patients with an LVAD implanted, the evaluation of the overall physical condition may be more subtle and suitable methods are required. As with other heart failure patients, cardiopulmonary stress tests $(4,5)$ and 6-minute walk tests $(6)$ are used for this purpose. These methods are also effective in LVAD patients, however they suffer several limitations. They provide only a partial picture of the patient physical condition at the time of the examination and do not reflect the broad range of conditions that an LVAD patient experiences during pump support. Moreover, LVAD patients suffering from neurological and physical deficits cannot usually undergo cardiopulmonary stress tests. Therefore, the overall estimate of the actual physical performance of the LVAD patient population might be overestimated due to the selection of "good" patients, able to perform such examinations (7). In addition to this potential bias, in LVAD patients it is not clear whether daily life activity correlates to the peak exercise capacity obtained from a cardiopulmonary stress test, as it is the case for heart failure patients (8).

A continuous, non-invasive, objective and cost-effective monitoring of the overall physical condition of the patient during everyday life activities may provide insights into the treatment, both during cardiac rehabilitation and daily life of LVAD patients. Such a monitoring can be realized using wrist-actigraphy (9-12). Wrist-actigraphy consists of an accelerometer implemented in a device similar to a wristwatch, which stores daily activity in minute intervals and allows ambulatory activity measurements with minimal impact on the quality of life of the patient. Actigraphy is a method that is gaining acceptance and it has been also used to objectively assess association between pain, activity and sleep quality, circadian rhythms and other patterns (e.g. activity vs. sedentary) in patients (9-14).

The aim of this study was to monitor daily life activity of LVAD patients from the early postoperative phase, during rehabilitation after initial hospital discharge and at home after rehabilitation discharge. Peak- $\mathrm{VO}_{2}$ values as an indicator for exercise capacity were correlated to daily life activity of LVAD and HTx patients. Additionally, daily life activity in LVAD recipients was compared to the activity measured in HTx and healthy matched subjects.

\section{Materials and Methods}

A prospective observational study was approved by the institutional ethical review board of the Medical University of Vienna and study participants provided informed signed consent. The study comprised a longitudinal part for thet investigation of daily life activity of LVAD recipients over the post-operative time course. Additionally, a cross-sectional part aimed at the comparison of daily activity of LVAD recipients to two additional groups: patients who underwent cardiac transplantation and healthy subjects. All the study participants were 
instructed to wear a wrist-accelerometer on the non-dominant arm and to keep the device continuously for the required duration without interruption unless any discomfort occurred. For the longitudinal part of the study, the main inclusion criterion was the implantation of a centrifugal-flow LVAD (HVAD, HeartWare Inc., Miami Lakes, FL, USA) for left ventricular support only. Patients were excluded if they had a different device implanted or biventricular support, if they could not wear continuously the wrist-accelerometer from study enrollment till the end of rehabilitation that followed hospital discharge (15), or if they could not participate in the first follow-up assessment. For the cross-sectional part of the study, the main inclusion criterion for both LVAD and HTx groups was that surgery occurred at least 12 weeks prior enrollment. Healthy subjects had matched characteristics to the LVAD recipients.

\section{Actigraphy}

Actigraphy data were collected using an Actiwatch II (Philips/Respironics, Murrysville, PA, USA). The Actiwatch II was configured to collect activity in 1-minute intervals. The device acquires activity by measuring the wrist acceleration and calculating a time series of the daily activity pattern using a digital integration algorithm. The measurement units provided by the device are arbitrary and are defined as an Activity Score, which is the result of the digital integration algorithm of the acceleration signal. Data were downloaded using the Actiware software (Philips/Respironics, Murrysville, PA, USA). Further analysis was performed using Matlab (The MathWorks, Natick, MA, USA), in particular daily life activity data were processed using Cosinor analysis (16). The Cosinor analysis provides a simple and standardized way to investigate circadian activities: In case of a variable that changes periodically during the course of the day a cosine wave is usually fitted to the time series. Activity during $24 \mathrm{~h}$ (from 0 to $24 \mathrm{~h}$ ) provided by the Actiwatch II represents such a variable. Therefore a cosine wave was fitted to the data (Figure 1) and the mean value of daily activity (so-called Mesor: midline estimation statistic of rhythm) and the difference between the peak and the mean value of the fitted cosine (Amplitude) were calculated.

\section{Time course of daily activity in LVAD recipients}

LVAD patients received the Actiwatch II after transfer from the intensive care unit to normal ward. The daily activity was further continuously recorded after hospital discharge during 4 weeks of rehabilitation, which all LVAD recipients undergo in our center (15). Following rehabilitation, the enrolled patients received the Actiwatch II for one week at two follow-up assessments approx. 3 and 7 months after hospital discharge (approx. 4.5 and 8.5 months after implantation) to monitor the time course of daily activity in LVAD outpatients. To test whether the mean activity at different time points are equal or not a one-way ANOVA (analysis of variance) for repeated measurements with Bonferroni correction was used.

\section{Comparison of daily activity in LVAD, heart transplanted patients and a healthy population}

To compare daily activity of LVAD recipients to the one of HTx patients and to a healthy population, study participants were enrolled in each of the three groups. In the LVAD group this included the patients followed longitudinally and, if required, additional ones just monitored for one week at about 4.5 months post implantation (to match the first follow-up in the longitudinal cohort). The HTx patients were chosen among those who had previously 
a LVAD implanted. Each subject in both groups received the Actiwatch II at least 12 weeks after surgery (pump implant or cardiac transplant). Healthy subjects were selected to match age, gender and BMI of the LVAD patient group. To compare the daily activity of the three different populations, the mean value of the Mesor and Amplitude of each patient was calculated for each group and compared using one-way ANOVA. The Tukey-Kramer post hoc test was then employed to compare all possible pairs of groups and provided correction for multiple testing.

\section{Comparison of peak- $\mathrm{VO}_{2}$ values and daily activity in LVAD and heart transplanted patients}

If patients from the LVAD and HTx groups underwent a cardiopulmonary stress test during cardiac rehabilitation, then the peak- $\mathrm{VO}_{2}$ values reached during this test were compared to the daily life activity measurements by the wrist-accelerometer. The Pearson correlation coefficient was calculated and regression analysis was performed.

\section{Results}

Between July 2012 and August 2013, 60 patients were implanted with a ventricular assist device at the General Hospital of Vienna. Among these, 36 patients had an HVAD implanted for left ventricular support only, 6 had biventricular assistance and 18 received another type of assist device. Among the 36 HVAD patients, 18 were excluded from the study for the following reasons: seven died within the first three months post implantation, four had psychological problems with associated poor compliance, two had early occurrence of adverse events, one never left the ICU and died at day 149, one was transplanted at day 78, one had a knee injury that impaired walking, one did not undergo rehabilitation, one refused to participate to the study. Of the remaining 18 patients, 11 took part to the longitudinal part of the study to investigate the time course of activity in LVAD patients. Among them one patient (Patient 4) was transplanted before the second follow-up and one patient (Patient 10) had to quit rehabilitation after 3 weeks due to gastrointestinal bleeding and hospital readmission, but could otherwise undergo the first follow-up. Since these two patients completed all other study related assessments, they were included into the analysis. The demographics of the 11 patients followed longitudinally are presented in Table 1.

\section{Time course of daily activity in LVAD recipients}

Mean daily activity increased significantly ( $55 \pm 28$ vs. $102 \pm 23$ Activity Score, $\mathrm{p}=0.002$ ) after initial hospital discharge to the first week of rehabilitation, respectively. During the 4 weeks of rehabilitation daily activity remained almost constant (102 \pm 23 vs. $112 \pm 34$ Activity Score, $\mathrm{p}=0.248$ ). At the follow-up assessments no significant change was observed compared to the activity at discharge from rehabilitation ( $112 \pm 34$ vs. $137 \pm 35$ Activity Score, $p=0.999)$. Mean daily activity did not change significantly from the first to the second follow-up, indicating a stable daily activity in outpatients ( $137 \pm 35$ vs. $148 \pm 35$ Activity Score, $p=0.999$ ).

Nevertheless, a trend towards increasing daily life activity with time could be observed during the study period (in hospital: $55 \pm 28$ Activity Score, rehabilitation week 4: $112 \pm 34$ Activity Score, second follow-up: $148 \pm 35$ Activity Score). Similar results were observed for the Amplitude of the daily activity (Figure 2). 
5 of the 11 included patients experienced at least one adverse event requiring hospital readmission during the study period. These patients showed no significant difference in activity at any of the assessed period compared to those without adverse events, except during the early postoperative period in the hospital (lower in patients without adverse events, $38 \pm 15$ vs. $75 \pm 27$ Activity Score, $\mathrm{p}=0.02$ ). A regular pattern in the activity over time was seen in 5 out of 6 patients without adverse events in contrast to an irregular activity pattern seen in 4 out of 5 patients suffering from adverse events. After an initial increase of daily activity following hospital discharge, which was seen in both groups, patients without adverse events had a stable activity during rehabilitation. This was followed by a substantial increase after rehabilitation discharge and a stable activity during the two follow-up assessments. In patients suffering from adverse events, more irregular patterns in the time course of daily activity were observed.

\section{Comparison of daily activity in LVAD, heart transplanted patients and a healthy population}

Patients included in the cross-sectional part of the study were 18 for each cohort. Among the three groups no statistically significant difference in age, gender and BMI was observed (Table 2). The post-operative day at examination (POD) was statistically different in the LVAD and HTx groups due to the rather low number of transplanted patients per year and therefore the inability to match this variable. LVAD patients were monitored a slightly longer period than the other two groups.

Mesor as well as Amplitude of daily activity was lowest in LVAD recipients, reaching 53\% of the healthy population. Although the daily activity was higher in HTx patients $(60 \%$ for Mesor and 65\% for the Amplitude compared to the healthy population), no significant difference was observed between the LVAD and HTx population. The healthy population was characterized by a significantly higher daily activity than the LVAD and HTx groups (Table 2 and Figure 3).

\section{Comparison of peak- $\mathrm{VO}_{2}$ values and daily activity in LVAD and heart transplanted patients}

15 Patients from the LVAD group and 13 from the HTx group underwent a cardiopulmonary stress test (at POD $73 \pm 26$ and $63 \pm 41$, respectively). Peak- $\mathrm{VO}_{2}$ was $9.7 \pm 2.5(34.4 \pm 7.9 \%$ of the expected value) in the LVAD and $10.5 \pm 4.9 \mathrm{ml} / \mathrm{min} / \mathrm{kg}(36.7 \pm 10.7 \%$ of the expected value) in the HTx population. Although the cardiopulmonary stress test and the daily life activity assessments were not performed in the same period, significant correlations were observed between activity and peak- $\mathrm{VO}_{2}$ (Figure 4). The Amplitude of daily life activity indicated a stronger correlation with peak- $\mathrm{VO}_{2}$ than Mesor (inset in Figure 4). 2 out of 3 included female patients had exceptional high Amplitude of daily life activity but a rather low peak- $\mathrm{VO}_{2}$ (Figure 4). Excluding female patients from analysis resulted in stronger correlations between peak- $\mathrm{VO}_{2}$ and daily activity (inset in Figure 4). Regression analysis indicated a slope of 13.9 Activity Score $/(\mathrm{ml} / \mathrm{min} / \mathrm{kg}$ ) without female patients and 11.8 Activity Score $/(\mathrm{ml} / \mathrm{min} / \mathrm{kg}$ ) for all patients (Figure 4). 


\section{Discussion}

In the current study physical activity was objectively measured by wrist-accelerometer in patients receiving a LVAD. The main finding from this study is the improvement of activity quickly after hospital discharge up to a plateau throughout 8.5 months post LVAD implantation. Adverse events make this activity pattern more irregular. A further finding of this study is that the level of activity at the plateau is comparable to activity in patients after a cardiac transplant but it reaches only the half of the activity measured in healthy control subjects.

Recently several studies investigated the physical performance of LVAD patients using bicycle spiroergometry to assess the peak- $\mathrm{VO}_{2}(4,7,15,17)$. These studies indicated limited exercise capacity of LVAD recipients with a peak- $\mathrm{VO}_{2}$ in the range of $50-60 \%$ of age and gender matched controls. Notwithstanding the importance of maximal exercise performance tests, these might not be the ideal tool for this patient population (7) and an objective measurement of the daily physical activity could help to better substantiate the patients' perceptions (7). Furthermore with such tests, the patient activity during the whole postoperative course and particularly in the out-of-hospital setting cannot be assessed. One of the strengths of this study addresses these limitations by the use of an objective measurement method to understand the effect of LVAD implantation on physical activity during the first 8.5 months after surgery. Within this observation window the impact of rehabilitation or the occurrence of adverse events on a patient's physical activity could be observed.

More recently, a study assessed daily life activity of LVAD, HTx and heart failure patients (18) by using actigraphy in a comparable way to the current study. A similar post-operative pattern of activity as well as an analogous limitation of LVAD patients compared to HTx and healthy subjects was reported. In (18), however, a different device to measure daily life activity was used. Our study confirms the findings reported in (18) and it shows that an objective measurement of activity can be obtained rather independently on the device used. A limitation of the actigraphy device used in the current study must be however acknowledged: some activities cause more prominent accelerations on the arm (e.g. walking) than others (e.g. biking) and the output of the device will not reflect the correct intensity of activity, especially, if one is interested in values related to energy expenditure. The patients in the HTx group were all chosen among previous LVAD recipients in order to observe how activity of a LVAD recipient changes after cardiac transplantation. It must be mentioned, however, that daily activity of patients who receive heart transplantation without being LVAD recipient could be different, even though the post-HTx survival is not different when comparing previous LVAD or conventional transplantation patients (19).

In addition to related findings as reported in (18), the current study presents three novel aspects: how the daily activity behaves during the structured rehabilitation training program that patients undergo in our center (15), the influence of adverse events on the time course of daily activity, and the correlation between peak- $\mathrm{VO}_{2}$ and daily activity.

Activity increases almost 2 -fold from the early postoperative period in the hospital to the period after hospital discharge; however, during the 4 weeks of rehabilitation activity 
increased only slightly. Although the constant value during rehabilitation seems odd at the first glance, it might be explained by the activities during rehabilitation scheduled rather evenly during the whole rehabilitation stay. After rehabilitation activity improved as measured at the two follow-up assessments; however, such improvement did not reach statistical significance compared to daily activity during rehabilitation.

Whether the plateau reached post-operatively was stable or not, heavily depended on the occurrence of adverse events. In this study it could be shown, in a limited number of patients, that the occurrence of adverse events is correlated to an instable activity pattern over time. This finding shows how accelerometers might become useful tools for screening, monitoring, feedback, and evaluation purposes for patients (20), also in consideration of the strong relationship between physical activity and cardiovascular health $(11,21)$. However, the small sample size limits the generalizability of the study. Furthermore, daily life activity was not measured continuously but only at given time points for the follow-ups. Therefore, the usefulness of such devices to monitor and even detect an early occurrence of adverse events could not be completely evaluated. Once the results presented are confirmed in a larger patient population, they will provide a valuable tool for a better tailored rehabilitation of LVAD patients.

In line with the findings in heart failure patients (8), in the presented study a significant correlation between the peak- $\mathrm{VO}_{2}$ values assessed during rehabilitation and daily activity measurements in LVAD as well as HTx patients could be identified, especially when only male subjects were included. Due to the small number of female subjects it remains speculative whether a gender related difference in the relationship between peak- $\mathrm{VO}_{2}$ and daily activity in LVAD and HTx patients actually exists. It can be speculated that these differences may be related to the different type of daily life activities in male and female.

\section{Conclusion}

In this study, an early improvement in activity post LVAD implantation was observed, which however reached a plateau during the observation period of 8.5 months. The level of activity achieved by the LVAD patients was just the half than control subjects would achieve, thus highlighting the need for an optimized physical therapy in this patient cohort.

\section{Acknowledgements}

The authors would like to thank Dr. Gorazd Sveticic and Dr. Zoltan Micskei from the "Department of Special Anesthesia and Pain Therapy, Medical University of Vienna, Vienna (A)" for their assistance with the actigraphy devices.

Financial support: This study was supported by the Austrian Science Fund (FWF): KLI357.

\section{References}

1. Kirklin JK, Naftel DC, Pagani FD, et al. Sixth INTERMACS annual report: a 10,000-patient database. J Heart Lung Transplant. 2014; 33(6):555-564. [PubMed: 24856259]

2. Klotz S, Jan Danser AH, Burkhoff D. Impact of left ventricular assist device (LVAD) support on the cardiac reverse remodeling process. Prog Biophys Mol Biol. 2008; 97(2-3):479-496. [PubMed: 18394685] 
3. Kobashigawa JA, Leaf DA, Lee N, et al. A controlled trial of exercise rehabilitation after heart transplantation. N Engl J Med. 1999; 340(4):272-277. [PubMed: 9920951]

4. Jakovljevic DG, George RS, Nunan D, et al. The impact of acute reduction of continuous-flow left ventricular assist device support on cardiac and exercise performance. Heart. 2010; 96(17):13901395. [PubMed: 20643664]

5. Balady GJ, Arena R, Sietsema K, et al. Clinician's Guide to cardiopulmonary exercise testing in adults: a scientific statement from the American Heart Association. Circulation. 2010; 122(2):191225. [PubMed: 20585013]

6. Foray A, Williams D, Reemtsma K, Oz M, Mancini D. Assessment of submaximal exercise capacity in patients with left ventricular assist devices. Circulation. 1996; 94(9 Suppl):II222-II226. [PubMed: 8901750]

7. Fabienne D, Hilde B, Bart M. To "pump up" quality of life and exercise performance studies in patients with left ventricular assist devices. J Heart Lung Transplant. 2011; 30(2):141-143. [PubMed: 21215379]

8. Jehn M, Schmidt-Trucksass A, Schuster T, et al. Daily walking performance as an independent predictor of advanced heart failure: Prediction of exercise capacity in chronic heart failure. Am Heart J. 2009; 157(2):292-298. [PubMed: 19185636]

9. Howell J, Strong BM, Weisenberg J, et al. Maximum daily 6 minutes of activity: an index of functional capacity derived from actigraphy and its application to older adults with heart failure. $\mathrm{J}$ Am Geriatr Soc. 2010; 58(5):931-936. [PubMed: 20374397]

10. Ancoli-Israel S, Cole R, Alessi C, Chambers M, Moorcroft W, Pollak CP. The role of actigraphy in the study of sleep and circadian rhythms. Sleep. 2003; 26(3):342-392. [PubMed: 12749557]

11. Paudel ML, Taylor BC, Ancoli-Israel S, et al. Rest/activity rhythms and mortality rates in older men: MrOS Sleep Study. Chronobiol Int. 2010; 27(2):363-377. [PubMed: 20370475]

12. Tranah GJ, Blackwell T, Ancoli-Israel S, et al. Circadian activity rhythms and mortality: the study of osteoporotic fractures. J Am Geriatr Soc. 2010; 58(2):282-291. [PubMed: 20374404]

13. Andrews NE, Strong J, Meredith PJ, D'Arrigo RG. Association between physical activity and sleep in adults with chronic pain: a momentary, within-person perspective. Phys Ther. 2014 Apr; 94(4): 499-510. [PubMed: 24231224]

14. English C, Manns PJ, Tucak C, Bernhardt J. Physical activity and sedentary behaviors in people with stroke living in the community: a systematic review. Phys Ther. 2014 Feb; 94(2):185-96. [PubMed: 24029302]

15. Marko C, Danzinger G, Kaeferbaeck M, et al. Safety and Efficiency of Cardiac Rehabilitation for Patients with Continuous Flow Left Ventricular Assist Devices. Eur J Prev Cardiol. 2014 Nov 7. [Epub ahead of print].

16. Nelson W, Tong YL, Lee JK, Halberg F. Methods for cosinor-rhythmometry. Chronobiologia. 1979; 6(4):305-323. [PubMed: 548245]

17. Kugler C, Malehsa D, Tegtbur U, et al. Health-related quality of life and exercise tolerance in recipients of heart transplants and left ventricular assist devices: a prospective, comparative study. J Heart Lung Transplant. 2011; 30(2):204-210. [PubMed: 20980169]

18. Jakovljevic DG, McDiarmid A, Hallsworth K, et al. Effect of left ventricular assist device implantation and heart transplantation on habitual physical activity and quality of life. Am J Cardiol. 2014; 114(1):88-93. [PubMed: 24925802]

19. John R, Pagani FD, Naka Y, et al. Post-cardiac transplant survival after support with a continuousflow left ventricular assist device: impact of duration of left ventricular assist device support and other variables. J Thorac Cardiovasc Surg. 2010; 140(1):174-81. [PubMed: 20447659]

20. Tudor-Locke C. Steps to Better Cardiovascular Health: How Many Steps Does It Take to Achieve Good Health and How Confident Are We in This Number? Curr Cardiovasc Risk Rep. 2010; 4(4): 271-276. [PubMed: 20672110]

21. Shiroma EJ, Lee IM. Physical activity and cardiovascular health: lessons learned from epidemiological studies across age, gender, and race/ethnicity. Circulation. 2010; 122(7):743-752. [PubMed: 20713909] 


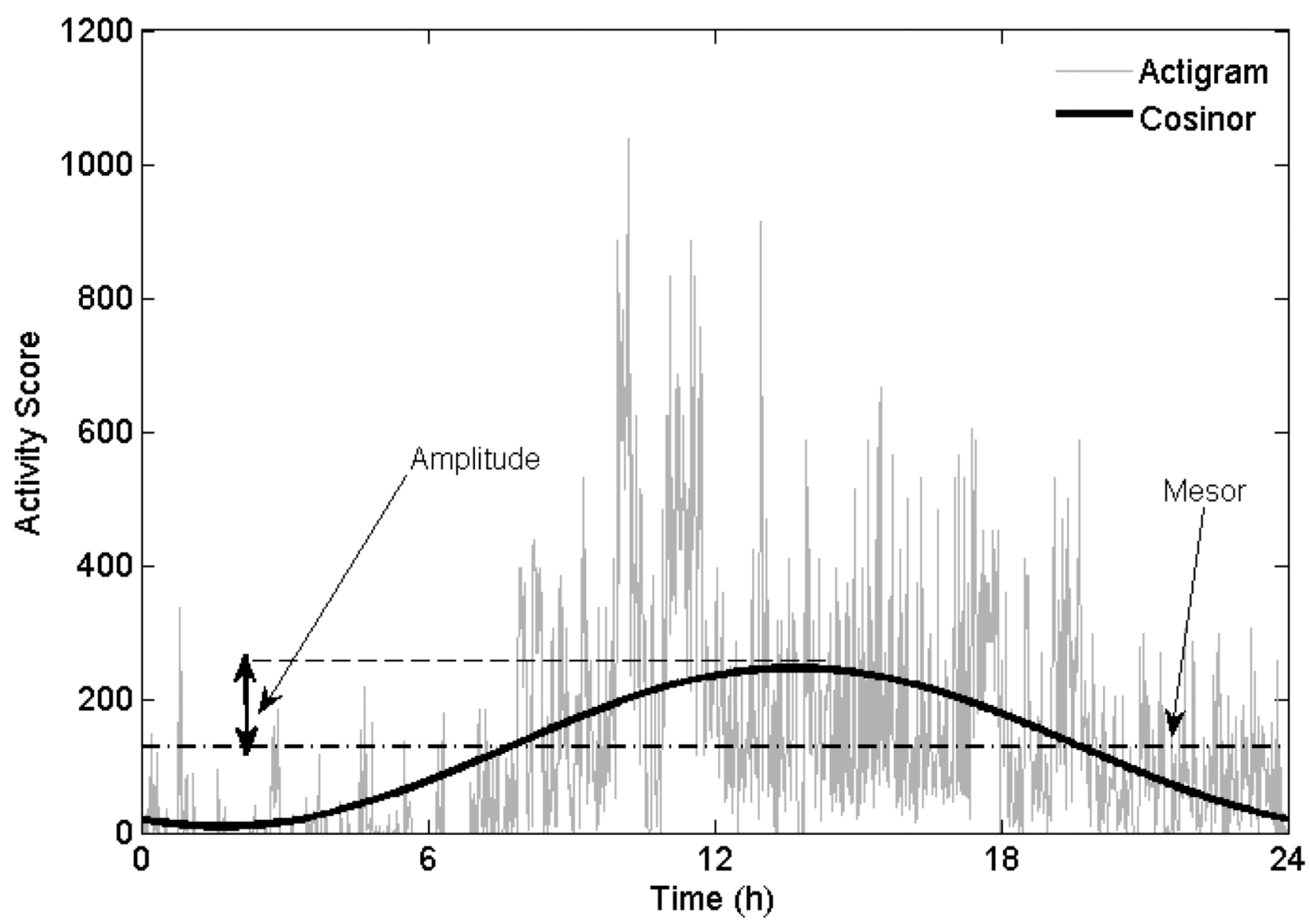

Figure 1.

The actigram (solid gray line) of a LVAD recipient during 24 hours. The fitted cosine wave (solid black line) as well as its mean value (Mesor, dash-dot black line) and difference between maximal and mean value (Amplitude, double-arrow) is indicated. The Mesor and the Amplitude were in this example 128 and 117 Activity Score, respectively. 

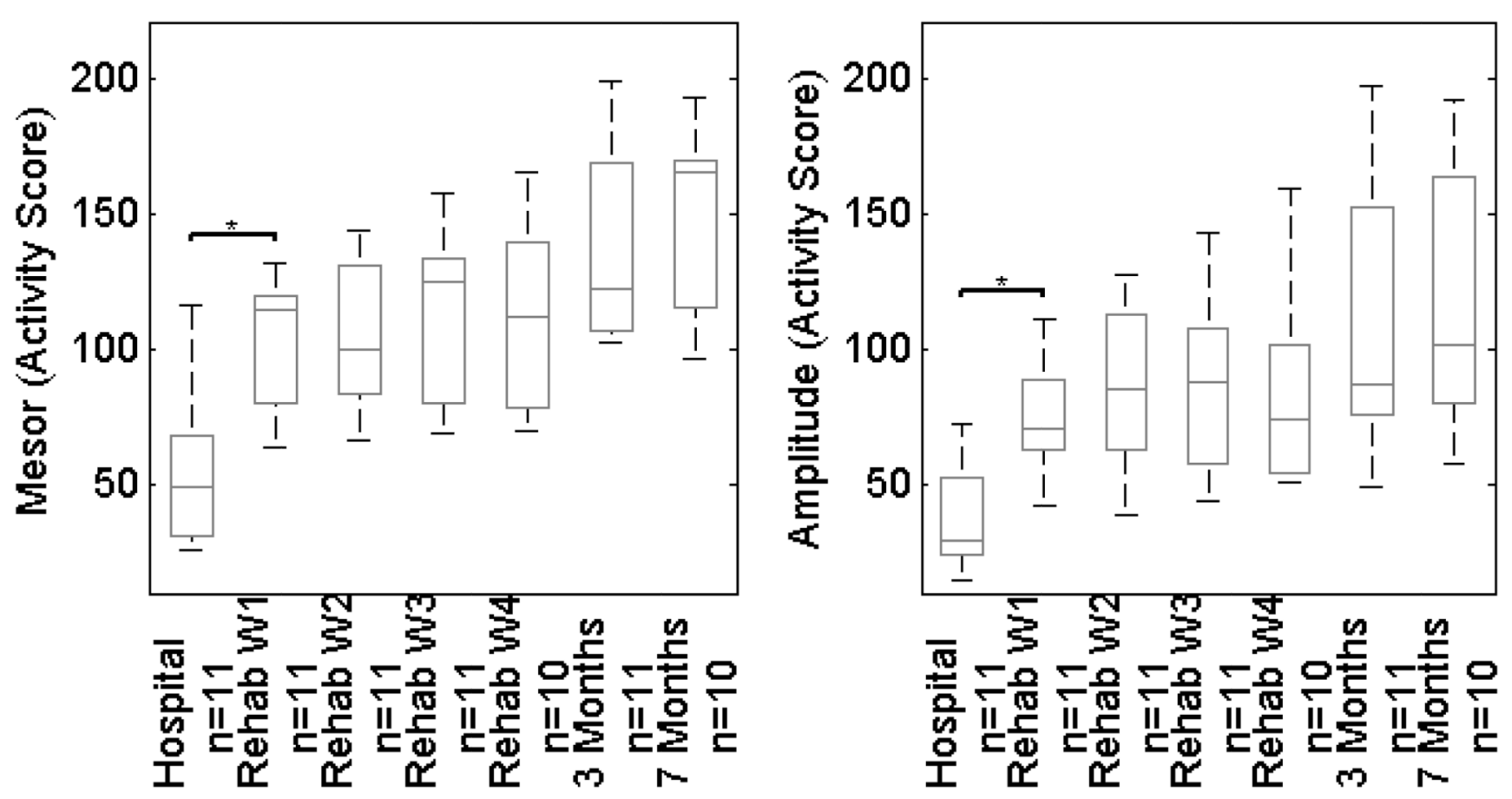

Figure 2.

Time course of daily activity of 11 LVAD patients assessed by the Mesor (left) and the Amplitude (right) of the actigram, during in-hospital stay, during 4 weeks of rehabilitation (W1 to W4) and at two outpatient follow-up examinations (month 3 and month 7). Activity changes significantly after hospital discharge $(* p=0.002$ for the Mesor and $p=0.012$ for the Amplitude) and tends to increase subsequently. For the deifinition of Mesor and Ampliture please refer to Figure 1. 

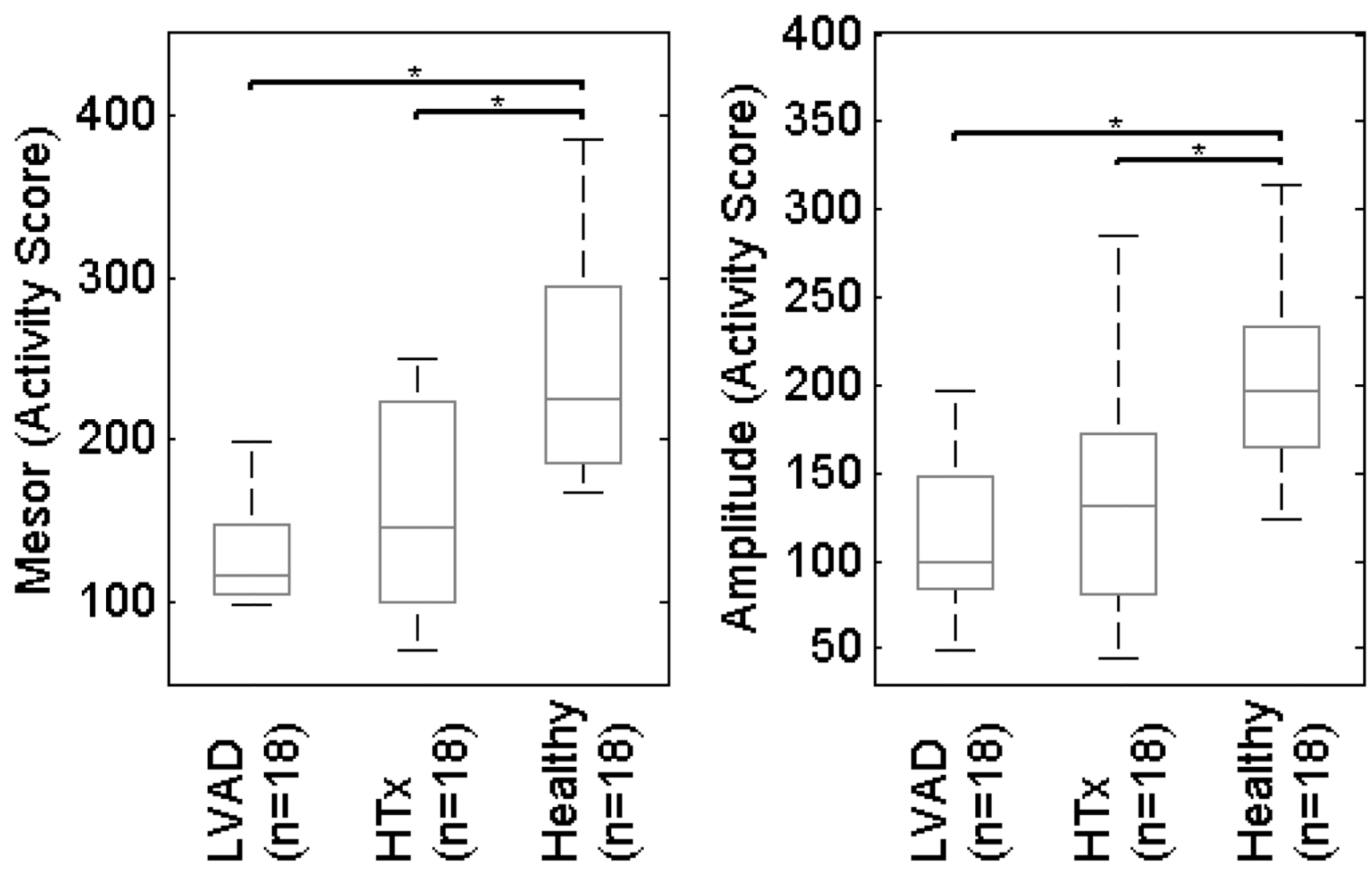

Figure 3.

Daily life activity for the three groups: patients with an LVAD, heart transplanted patients (HTx) and healthy subjects. Activity is quantified by the Mesor (left) and the Amplitude (right). LVAD and HTx patients show comparable activity, in both cases significantly lower than in healthy matched subjects ( $* \mathrm{p}<0.001$ for all comparisons). For the deifinition of Mesor and Ampliture please refer to Figure 1. 


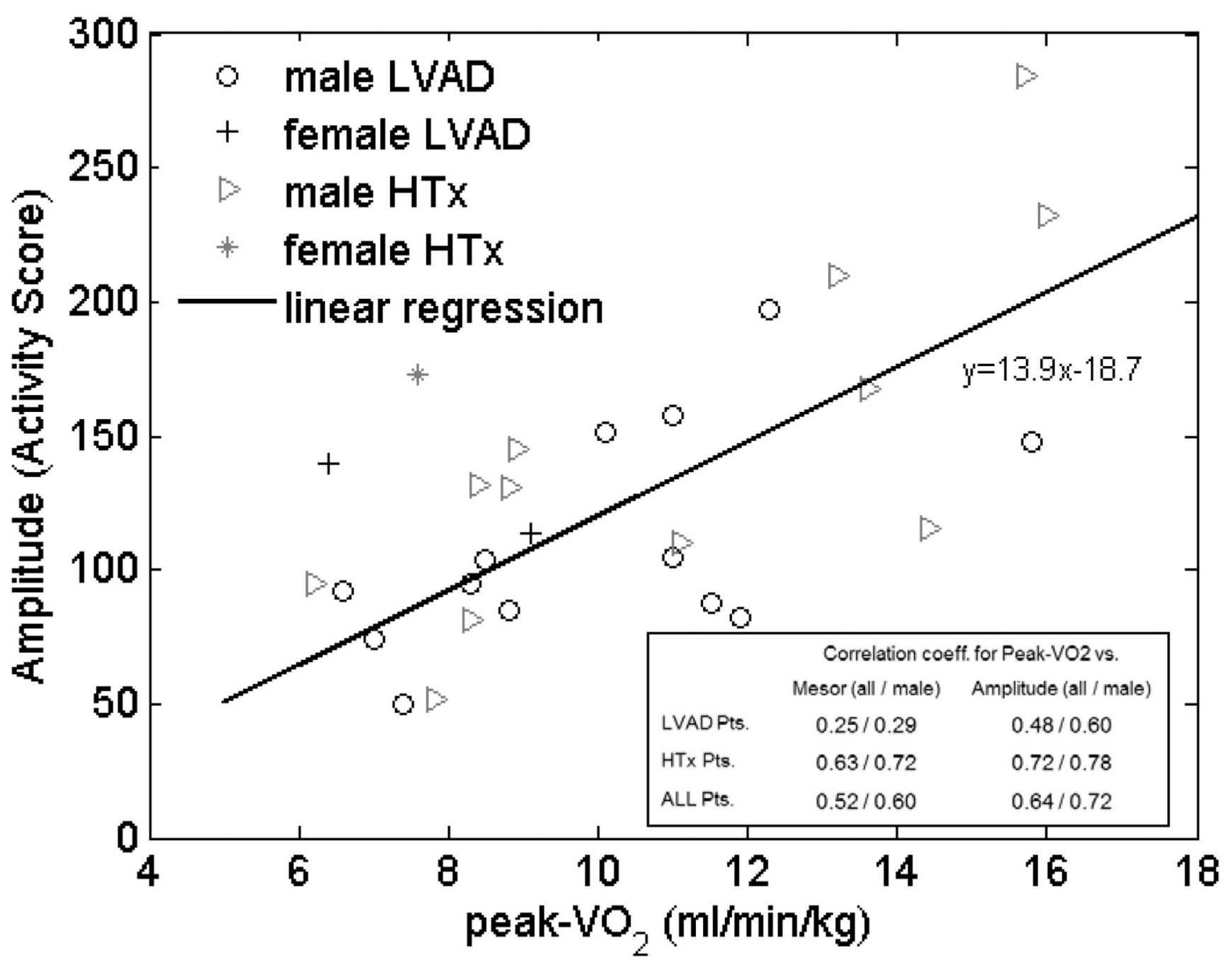

Figure 4.

Relationship between peak oxygen consumption (peak- $\mathrm{VO}_{2}$ ) and the Amplitude of daily activity in the LVAD population (circle and cross symbols) and the heart transplanted (HTx) population (triangle and asterisk symbols). Male and female subjects are denoted by different symbols. The black line indicates the linear regression for male patients alone. In the inset the correlation coefficients are shown between peak- $\mathrm{VO}_{2}$ values and parameters of daily activity for LVAD population ( $n=15)$, HTx patients $(n=13)$ and the combination of both groups $(n=28)$. Correlation tends to be higher in male patients. For the deifinition of Mesor and Ampliture please refer to Figure 1. 


\section{Table 1}

Demographics of the 11 LVAD patients in whom the time course of daily life activity was assessed. Data are expressed as mean \pm standard deviation. Ranges are given in square brackets.

\begin{tabular}{cc}
\hline Age (years) & $55.4 \pm 9.4[33-69]$ \\
\hline BMI $\left(\mathrm{kg} / \mathrm{m}^{2}\right)$ & $26.1 \pm 3.2[20.8-31.0]$ \\
\hline Gender (male/female) & $10 / 1$ \\
\hline Intermacs level (1/2/3/4) & $3 / 1 / 6 / 1$ \\
\hline POD at rehabilitation admission & $41 \pm 23[20-108]$ \\
\hline POD at first follow-up & $140 \pm 43[85-256]$ \\
\hline POD at second follow-up & $253 \pm 33[206-300]$ \\
\hline
\end{tabular}

BMI: Body Mass Index; POD: post operative day 


\section{Table 2}

Demographics and comparison of out-of-hospital activity of the three groups: patients with an LVAD, heart transplanted patients and healthy subjects. Data are expressed as mean \pm standard deviation. Ranges are given in square brackets.

\begin{tabular}{|c|c|c|c|c|}
\hline & LVAD (n=18) & HTx $(n=18)$ & Healthy $(n=18)$ & Significance \\
\hline Age (years) & $\begin{array}{c}57.6 \pm 10.5 \\
{[33-76]}\end{array}$ & $\begin{array}{c}54.9 \pm 8.6 \\
{[39-70]}\end{array}$ & $\begin{array}{c}54.9 \pm 10.5 \\
{[33-78]}\end{array}$ & $\mathrm{p}=0.661$ \\
\hline Gender (\%male) & 83.3 & 88.9 & 83.3 & $\mathrm{p}=0.739$ \\
\hline BMI & $\begin{array}{c}26.0 \pm 3.1 \\
{[18.6-32.5]}\end{array}$ & $\begin{array}{c}26.0 \pm 3.7 \\
{[19.0-30.5]}\end{array}$ & $\begin{array}{c}25.8 \pm 2.8 \\
{[22.1-32.9]}\end{array}$ & $\mathrm{p}=0.985$ \\
\hline POD & $\begin{array}{l}148 \pm 59 \\
{[86-328]}\end{array}$ & $\begin{array}{c}346 \pm 247 \\
{[99-883]}\end{array}$ & --------- & $\mathrm{p}=0.002$ \\
\hline Monitored days & $7.3 \pm 2.5$ & $5.7 \pm 1.8^{*}$ & $5.8 \pm 1.4^{*}$ & $\begin{array}{c}* \mathrm{p}=0.012 \\
\text { compared to LVAD }\end{array}$ \\
\hline $\begin{array}{l}\text { Mesor } \\
\text { (Activity Score) }\end{array}$ & $130 \pm 30^{*}$ & $148 \pm 60^{*}$ & $245 \pm 63$ & $\begin{array}{c}* \mathrm{p}<0.001 \\
\text { compared to healthy }\end{array}$ \\
\hline $\begin{array}{l}\text { Amplitude } \\
\text { (Activity Score) }\end{array}$ & $110 \pm 39^{*}$ & $135 \pm 68^{*}$ & $207 \pm 53$ & $\begin{array}{c}* \mathrm{p}<0.001 \\
\text { compared to healthy }\end{array}$ \\
\hline
\end{tabular}

LVAD: left ventricular assist device; HTx: heart transplant; BMI: body mass index; POD: post operative day. For the deifinition of Mesor and Ampliture please refer to Figure 1. 